\title{
Parametric Modeling of Archaeological Heritage in the Age of Digital Reconstruction
}

\author{
> Guzden Varinlioglu \\ University of California Los Angeles, USA \\ guzdenv@gmail.com \\ > Ozgun Balaban \\ Singapore University of Technology and Design, \\ Singapore \\ ozgunbalaban@gmail.com
}

> Yekta Ipek

Istanbul Technical University, Turkey

yektaipek@gmail.com

> Sema Alacam

Istanbul Technical University, Turkey

semosphere@gmail.com

\begin{abstract}
Although 3D modeling has become standard within archaeology, the process of digitally reconstructing archaeological objects requires clumsy and time-consuming procedures. Typically, these procedures call for careful collaboration, between architectural historians, archaeologists and computer modelers, to produce scientifically correct and artistically pleasing models. This paper proposes the use of shape grammars for digital reconstruction of the archaeological heritage artifacts that were discovered during surveys along the coast of Turkey. Concentrating on ceramic vessels, the archaeologists and divers classified the artifacts based on their dimensions and observations. The classifications are used to infer the rules that will generate the digital model for each artifact. Rhinoceros modeling software with Grasshopper plug-in was used to generate 3D models. The goal of this work is to demonstrate that knowledge generated within a virtual world experience, can inform the evaluation of data derived from textual and archaeological sources, and vice versa.
\end{abstract}

Keywords: Parametric modeling, Underwater cultural heritage, Grasshopper, 3D reconstruction.

\section{Introduction}

The research described in this paper is part of the 'Virtual Museum of Underwater Cultural Heritage' that contains a digital repository framework of underwater cultural heritage, to document, research and preserve underwater sites on the Mediterranean coast of Turkey. To create this framework, a novel data collection methodology for underwater archaeology surveys was designed. Without disturbing the archaeological heritage, divers recorded pertinent dimensions and shape data by populating structured data sheets. Categories, based on artifact shape and form, helped us develop shape grammars for the archaeological artifacts, in this case ceramic vessels. This dimensional and shape data set, together with sketches and photographs, were uploaded to a purpose-built MySQL database system. In order to streamline classic archaeological documentation, our objective was to create rules for documenting 3D non - geometric artifacts, driven by the shape grammar of artifacts found.

Reconstruction of archaeological artifacts from textual and metric data is typically a tedious task requiring many hours of work for archaeologists, architectural historians and computer modelers. However, $3 \mathrm{D}$ visualization of artifacts provides valuable information about the artifact for archaeologists to further conduct research and analysis, while also providing a visually pleasant presentation method for the general public. In his outdated but seminal article, Reilly (1992) presents two terms on archaeology in the information age: data visualization and solid modeling. Data visualization, with its connotations in advanced computing, refers to techniques which allow "visual interpretation of data through the representation, modeling and display of solids, surfaces, properties and animations, involving the use of graphics, image processing, computer vision and user interfaces" (Reilly, 1992: 147). During the 'Virtual Museum' project, we attempted a by-pass of the interim tasks between data collection and 3D model analysis.

The main motivation for the paper is to create a dialogue between the archaeologist in the field, as data collector, and the archaeologist on the computer, as analyst, through $3 \mathrm{D}$ models of the objects. The specific goals of this paper are twofold: first, to determine the shape grammar of the archaeological artifacts and second, to use computational tools to enable the archaeologists to have instant 3D models of the artifacts for analysis and interpretation.

Based on our systematic data collected during the surveys, and the structure of the database system, the shape grammar is determined to classify and analyze the ceramic pieces. The project foresees the development of grammars encoding the rules for reconstructing virtual ceramic vessels. This paper presents an excerpt of a preliminary version of the grammar for these ceramic vessels, focusing on Amphoras, and describes the computer implementation of the grammar, using Rhino and Grasshopper, after being converted into parametric design units and subsequent online presentation methods as produced from the output of the program. The models are included in the website of the main project 'Virtual Museum of Underwater Cultural Heritage'.

\section{Methodology}

The aim of archaeology is to study and relate an understanding of cultural heritage, based on how and where the remains are found. 
Cultural artifacts detached from their original context, are either transformed to a museum or, when kept in their original place, can deteriorate by natural forces or general neglect (Kalay, 2008: 1). Hence, a technology-driven alternative to preserve cultural heritage has emerged through the advances in new media. Researchers, societies, museums, universities, and governments have embraced the modeling and visualization abilities of computers to create reconstructions and databases of cultural heritage. A good example is the Roman Amphorae Project, an online introductory resource for the study of Roman amphoras; pointed two-handled ceramic vessels used for carrying and keeping various supplies, primarily wine and oil. Supported by the University of Southampton, the Archaeology Data Service (ADS), concentrates on the principal types of ceramic vessels manufactured throughout the Roman Empire (Keay, 2005). The aim of the site is to provide basic information about the more common types of amphoras while trying to catalogue and categorize as broad a geographical spread as possible. In other words, this project uses shape grammar to describe the known Roman amphoras, based on previous archaeological studies and findings.

Shape grammar (Stiny, 1980) is a formal method of computation that addresses the shape and geometric form of artifacts, and is valuable as a descriptive, interpretative, generative and evaluative tool in analysis and design. With a set of rules and vocabulary of shapes, shape grammars can describe and generate possible designs. The visual representation of data allows the archaeologist to evaluate its validity or identify discrepancies more easily than with symbolic and verbal descriptions. Grammars and shape grammars are not new to archaeological research. Knight authored a grammar to analyze the Maender motif in Greek Geometric Pottery and show how changes in the grammar account for stylistic change based on temporal/ geographic criteria (Knight, 1986). Coutinho et al. authored a paper in eCAADe entitled 'A computational interpretation of 'De re aedificatoria '", translating Alberti's column system into a shape grammar (Coutinho et al., 2011). In the study of Digital Alberti, the cultural impact of Alberti's treatise on Portuguese classical architecture is studied using a computational approach. The treatise is translated into a shape grammar i.e. into a parametric modeling system and finally into a computer program to analyze and generate the column system (Coutinho et al., 2011: 800).

These projects emphasize various possibilities of analysis, and methods of visualization, using structured data in archaeology and architecture. The structured data defining the parts of the artifacts can be thought of as a set of algorithms that explain how to reconstruct the $3 \mathrm{D}$ artifacts. Our aim is to translate this data into a shape grammar using procedures similar to Couthinho. As per the Couthinho approach, the procedure of our study encompasses the following four tasks: decoding artifacts, inferring grammar, implementing grammar and generating digital models.

\section{Decoding the artifacts}

An integral part of the 'Virtual Museum of Underwater Cultural Heritage' saw the underwater archaeologists identify and categorize amphoras according to their physical properties and size. In the case of ceramic vessels, descriptions of rim, neck, handle, shoulder, base

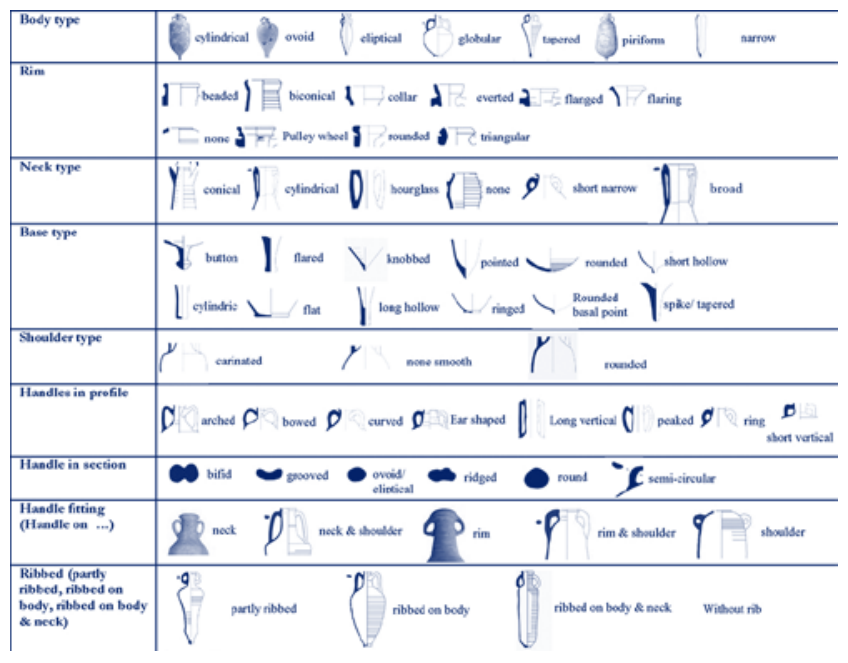

Fig. 1: Example of a shape grammar chart of amphoras

and body types were separately documented, in addition to height, width, rim, neck and base diameters, as well as shoulder and handle heights. In certain cases, further details such as handle section, or observations made on body decorations and ribs, were also included (Fig.1) and stored in the MySQL database (Varinlioglu, 2012).

Although a large range of artifacts were documented, including large ceramic vessels and pithoi, stone and stock anchors, ballast stones, millstones and other architectural cargo, for the purpose of this paper the focus is on amphoras. The amphora types differ greatly in shape and size depending on where and when they were produced. Amphoras were traded throughout the ancient Mediterranean and transported as cargo material over long distances. As symbols of maritime trade, these vessels support clues for dating and defining the origin of the archaeological remains. Various dating systems and theories are based on catalogues depicting their production center known through on-land excavations. The transportation of amphoras through the marine trades gives insight into the possible trade connections between cultures and territories.

\begin{tabular}{|l|l|}
\hline Length $(\mathrm{cm})$ & 55 \\
\hline Width $(\mathrm{cm})$ & 37 \\
\hline Width shoulder $(\mathrm{cm})$ & 25 \\
\hline Ceramic thickness $(\mathrm{cm})$ & 2 \\
\hline Rim diameter $(\mathrm{cm})$ & 5 \\
\hline Rim thickness $(\mathrm{cm})$ & 1 \\
\hline Neck height $(\mathrm{cm})$ & 10 \\
\hline Neck diameter max. $(\mathrm{cm})$ & 15 \\
\hline Neck diameter min. $(\mathrm{cm})$ & 4 \\
\hline Handle height $(\mathrm{cm})$ & 14 \\
\hline Handle distance to body $(\mathrm{cm})$ & 7 \\
\hline Handle diameter $(\mathrm{cm})$ & 2 \\
\hline Handle thickness \& width $(\mathrm{cm})$ & $2 \& 4$ \\
\hline Base length $(\mathrm{cm})$ & 4 \\
\hline Base diameter, ring flat bottom $(\mathrm{cm})$ & 5 \\
\hline Thickness base & 2 \\
\hline
\end{tabular}

Table 1. Typological measurements on amphora KE-23-A. 


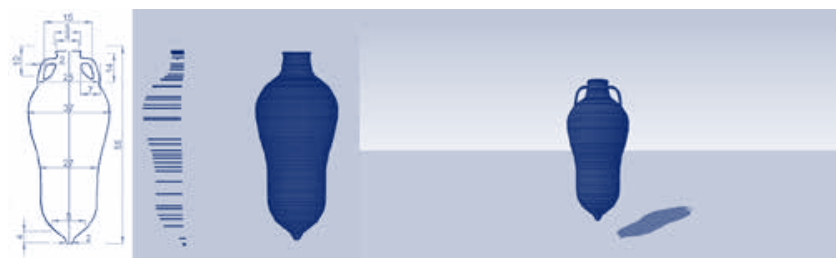

Fig 2. Steps for manually create $2 \mathrm{D}$ and $3 \mathrm{D}$ of the amphora KE-23-A

Based on these characterizations, inspired by the Roman Amphorae Project, each amphora is decoded by the parameters defining its shape (Varinlioglu, 2011: 186). The following table depicts an example of the artifact KE-23-A and its parameters, referred to as typological data in archaeological terms. The measurements, defining the overall dimensions, as well as detailed dimensions of the parts are also defined to help scale the typological data. This task is illustrated in Table 1 and is aimed at gaining a deeper understanding of the parameters, described in the data sheets, by systematizing the collected data of the amphora, developing 2D drawings and $3 \mathrm{D}$ models of the artifacts (Fig 2.).

\section{Inferring the grammar}

This procedure is aimed at gaining a deeper insight into the shape and scale of amphoras, by using the described rule system, to translate them into shape grammars. For this, there are three basic steps. The first step is to create a diagram that captures the parts of the amphora and describe the formal structure of the geometry. The second step is to extract the rules for defining the characteristics of the various amphora parts and, the third step draws the shape grammar rules. The rules for making the $3 \mathrm{D}$ models and detailing the parts are described in the Table 2.

The 3D model of an amphora can be reduced to two main steps. The body type is the first generative clue about the form of the amphora, and the handle type is the second main component. The handles are later added to the main body. After the body type is selected, the parts of the amphora such as rim, neck, base and shoulder, are selected according to the rules. The cross-section of the handles, and where they are placed, are defined both by the typological data and the measurement data.

Shape grammars start with the first rule, which defines the overall object height, ObjHeight. The next rule divides this length into the three main parts of the amphora, that is: neck, body and base. At this stage, each of the parts is operated upon independently. For example, as is the case with amphora KE-23-A, if we are dealing with a beaded rim (one of many rim type options) it starts from the top and then draws the rim according to the parameters. To acquire the rules for specific object parts, the first step is to draw the idealized shapes for various types, and the second step is to drive the rules from the boundary curves. For example, a beaded rim has two different curves defining the final shape and respectively, the parameters of neck height and rim diameter determine its final shape. For rims, the curves are sketched on top, and are replicated with the new values such as the width and height of the rim.

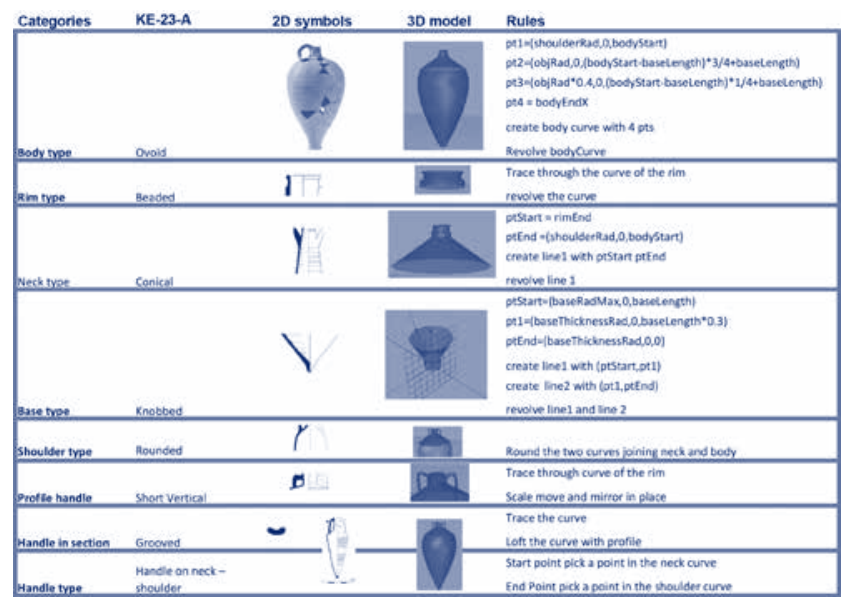

Table 2. Workflow for generating 30 model of the amphora KE-23-A

Other curves are digitized by taking the ratios from predetermined points that are subsequently uploaded to the Python script function of Grasshopper. As in the case of KE-23-A amphora, an ovoid body uses the objRad value to define the radius of the amphora at its widest. In order to draw the body, the curve passes through (objRad, $0,0.5^{*}$ bodyLength) which means halfway in the body height the curve attains its widest position whereas, in the end and start of the curve, the function reverts to shoulderRad or baseRad, respectively.

\section{Implementing the grammar}

This task deals with writing the computer program for encoding the grammar after converting it into a parametric modeling system. This conversion was straightforward because the grammar is a parametric set grammar. The computer implementation was developed using Rhino software with Grasshopper plug-in. This implementation permitted us to test the adequacy and accuracy of the grammar in conveying the rule system. It also permitted us to explore the system in the generation of 3D modeling of artifacts and to compare with their relevant sketches and photographs.

Python components within Grasshopper make the process neater as it is easier to create algorithmic structures of models in a programming language that simplifies NURBS geometry manipulation. Using Slingshot (plug-in developed for Grasshopper) the database is easily accessed. After acquiring the data, the objects are modeled within this environment and are again saved to the database.

\section{Generating Digital Models and Displaying the 30 Web Environment}

The final step in the methodology is the generation of digital models to share with online users in a $3 \mathrm{D}$ web environment. Javascript offers the opportunity to show 3D models in the web browser and to create visual and virtual environments. Specifically, using Javascript capabilities to render 3D models in the web browser, we provide end users with the ability to navigate interactively in a virtual environment. 


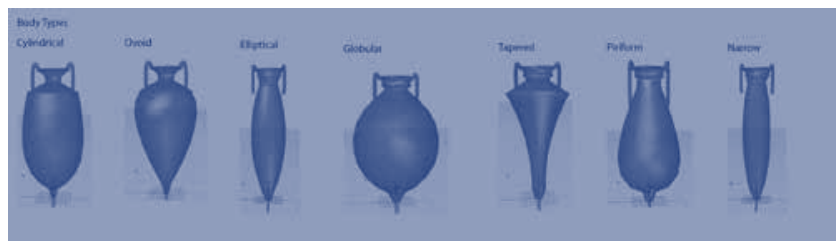

Fig. $\exists$ Generated models of various body types of amphora

ThreeJs javascript libraries are used to render the 3D models. Employing these libraries make it is possible to create virtual environments composed of different lighting types and background spaces. However, 3D models are rendered with a generic texture and color (as a ceramic piece), as underwater artifacts provide no data about their original material and color of the artifacts. Having created the background environment, the 3D models can then be embedded to the environment. Moreover, it is also possible to create a camera that can be controlled, by the user's mouse movements, to navigate around the $3 \mathrm{D}$ models.

To use the 3D model data within ThreeJs library, each 3D model generated by the data acquired from the database should be converted into OBJ file format; a universally accepted 3D model file format. These file formats also support NURBS surface as well as Polygonal Mesh surfaces. In accordance to the geometric data, the file format can carry the texture coordinate data and render the material textures as needed. Each generated 3D model is saved in OBJ file format with the texture data, where applicable.

In the web page template, pages are created with basic lightning and environments. Further to the creation of these environments, 3D models can be called to the scene on the web page. Without refreshing the page, other models can be called into the scene, and navigated. The model on the scene can be swapped with other models in accordance with end user's requirements.

With the use of the ThreeJS javascript library, 3D geometrical and textural data can be shown on the web browser with simple HTML pages. Thus, 3D models can be navigated on the web browser by the end user.

\section{Results}

Preliminary studies on the reconstruction of ceramic pieces produced accurate 3D solid models. Based on the measurements and typological data, the $3 \mathrm{D}$ modeling is proving to be a valuable research and analysis tool for archaeologists. The test results of the parametric modeling work flow proved the initial success for the method devised (Fig. 3). It is expected that the workflow will contribute to the knowledge formation process in digital archaeology, by automating model reconstruction when the automation and singular computer applications talk to each other.

\section{Conclusion}

Recent developments in parametric modeling brought challenges in digital reconstruction of ancient archaeological sites and objects. In this paper, we present a workflow for the $3 \mathrm{D}$ reconstruction of cultural heritage artifacts through the use of Grasshopper and parametric modeling. Our workflow begins by defining shape grammars of artifacts found along the Mediterranean coastline of Turkey. Focused on ceramic pieces, the 3D models of archaeological objects are parametrically reconstructed based on data provided in a database system specifically designed for the archaeological survey project. The parametric modeling system takes the data from the database and creates $3 \mathrm{D}$ models and offers 3D information instantly to archaeologists. Used both as a research and visualization tool, this work flow introduces an automatic method of digital reconstruction of archaeological artifacts.

\section{Acknowledgements}

This work comes within the context of research conducted as part of Varinlioglu 's postdoctoral research at Istanbul Technical University and University of California Los Angeles. Balaban accomplishes the computational part during the Design Computation in Architecture course, which is taught by Bige Tuncer at Singapore University of Technology and Design. Lastly we are indebted to Gulen Cagdas and Bige Tuncer for their critiques on the computational aspects of the project, to Diane Favro and Christopher Johanson for support on Varinlioglu's post-doctoral research in UCLA.

\section{References}

Coutinho, F., Costa, E. C., Duarte, J. P. \&,Kruger, M. (2011). A computational interpretation of "De re aedificatoria": Translating Alberti's column system into a shape grammar. eCAADe 29 Shape Studies, 799-809.

Kalay, Y. E. (2008). Preserving cultural heritage through digital media. In Y. E. Kalay, T. Vvan, \& J. Affleck (Eds.) New Heritage: New Media and Cultural Heritage (1-10). London and New York: Routledge.

Keay, S. (2005). Roman Amphorae: a digital resource. Retrieved from http://ads.ahds.ac.uk/catalogue/resources.html?amphora2005.

Knight, T. W. (1986). Transformations of the Meander Motif on Greek Geometric Pottery. Design Computing. 1, 29-67.

Reilly, P. (1992). Three-dimensional modelling and primary archaeological data. In P. Reilly \& S. Rahtz (Eds.), Archaeology and the Information Age: a Global Perspective (147-173). London, New York: Routledge.

Stiny, G. (1980). Introduction to Shape and Shape Grammars, Environment and Planning B 7 (3), 343-352.

Varinlioglu G. (2011). Data Collection for a Virtual Museum on the Underwater Survey at Kas, Turkey. International Journal of Nautical Archaeology 40: 1, 182-188.

Varinlioglu G. \& Ozguc B. (2012). Web Information System for Virtual Museum of Nautical Archaeology. SIGRADI 16, 204-208. 